\title{
The Effects Community Tuberculosis Care Makes in the Early Identification and Referral of Tuberculosis Patients from Active Case Findings in Botswana
}

\author{
Unami Mathebula-Modongo ${ }^{1 *}$, Mary Moleki ${ }^{1}$, Tefera Agizew $^{2}$ \\ ${ }^{1}$ Faculty of Health Sciences, University of South Africa, Pretoria, South Africa \\ ${ }^{2}$ Department of Family Medicine and Public Health, Faculty of Medicine, University of Botswana, Gaborone, Botswana \\ Email: `58536620@mylife.unisa.ac.za
}

How to cite this paper: Mathebula-Modongo, U., Moleki, M. and Agizew, T. (2021) The Effects Community Tuberculosis Care Makes in the Early Identification and Referral of Tuberculosis Patients from Active Case Findings in Botswana. Journal of Tuberculosis Research, 9, 230-244. https://doi.org/10.4236/jtr.2021.93022

Received: May 5, 2021

Accepted: September 27, 2021

Published: September 30, 2021

Copyright ( 2021 by author(s) and Scientific Research Publishing Inc. This work is licensed under the Creative Commons Attribution International License (CC BY 4.0).

http://creativecommons.org/licenses/by/4.0/ (c) (i) Open Access

\begin{abstract}
Setting: Twenty-two public health clinics offering Tuberculosis (TB) care at Francistown and Gaborone cities in Botswana. Objectives: To assess the effects of community tuberculosis care (CTBC) on the early identification and referral of patients and to identify perceived factors that contribute to effective active case findings and community referral system. Design: We adopted a concurrent triangulation mixed-method research design. Convenient purposive sampling was followed for both qualitative and quantitative phases. Data were collected from participants who met the inclusion criteria in both phases from July 2016-Dec. 2018. Consent was obtained from the all eligible participants. Results: We interviewed 40 caregivers with mean age of 43.4 years. We identified different themes, $83 \%(n=33)$ accepted the caregiving role, $50 \%(\mathrm{n}=20)$ reported Social and physiological support from health workers, $23 \%(\mathrm{n}=10)$ received health education on TB, $20 \%(\mathrm{n}=8)$ and $30 \%$ $(\mathrm{n}=12)$ reported teamwork and communication respectively between the health workers and caregivers as factors that contribute to effective active case findings in СТВС. 670 patients were enrolled in the quantitative phase and only $55 \%(n=369)$ had their contact details documented in the patient's TB records, while $94 \%$ (1311/1398) active case finding suspects/contacts screened for TB. Only 18\% (115/1398) had been referred for investigated and 3\% (35/ 1398) were TB positive. All patients underwent treatment. Conclusion: The caregiving role has been accepted in СТВC. Moreover, effects of CTBC on the early identification and referral of TB patients are commendable and can be improved.
\end{abstract}


Keywords

Caregiving, Tuberculosis, CTBC, Triangulation Study, Public Health

\section{Introduction}

Tuberculosis epidemic is amongst the leading causes of death worldwide. The World Health Organization (WHO) estimated that 10 million people developed tuberculosis (TB) and 1.5 million of these people died from the disease globally in 2018 [1]. The WHO has set certain milestones to end the global TB epidemic by reducing TB deaths from $35 \%-57 \%$ by 2020 and 2025 respectively [1]. In 2018 WHO reported that African region accounted for $25 \%$ of the TB cases [2]. Considering the aforementioned milestones and the high estimated incidence rates of 275 per 100,000 TB in Botswana [3], the Botswana TB program intended to reduce TB incidence using all strategies including community TB care (CTBC) as recommended by WHO since 2004 [3] [4] [5]. In addition, it aimed to meet the WHO-formulated "End TB strategy 2016-2035" "targets of reducing TB deaths by $95 \%$ and TB incidence by $90 \%$ " [2] [6]. This necessitates an early identification of TB cases and treating more people with active TB.

The CTBC in Botswana had experienced several challenges, some of which included the inability to report the community contributions to TB case notifications and the lack of support at all levels of authority [7]. The mission of the Botswana National TB Program Strategic Plan 2013-2017 was to reduce TB incidence and prevalence such that it will no longer be a public health problem. This called for the need to assist the Ministry of Health and Wellness (MOHW) to reduce the incidence and prevalence of TB in Botswana [8]. It is crucial to identify the CTBC referral system and effective factors that contribute to the community in TB active case findings such that effective contributions are scaled up to promote quality care since it is the first investigation of its kind carried out in Botswana and regionally. This in turn would facilitate meeting the WHO set targets in TB diagnosis and treatment outcomes in Botswana. We aimed to assess the effect of CTBC on the early identification and referral of TB patients from active case finding and to identify what the community perceived factors that contribute to effective active case findings and a community referral system.

\section{Design}

We adopted a concurrent triangulation mixed-method research design (both qualitative and quantitative). We conducted a descriptive qualitative research to identify what the community perceived factors that contribute to effective active case findings and the community referral system. In contrast, we conducted a descriptive quantitative research to assess the effects of CTBC on the early identification and referral of patients with TB from active case findings. Written consent was obtained from the affected institutions and eligible participants. 


\subsection{Setting and Sampling Methods}

We selected Francistown and Gaborone as the study sites because they are the two major cities of Botswana, one on the north and other in the south side of the country. Gaborone ad Francistown has a population of 231,592 for Gaborone and 98,961 for Francistown [9]. African Comprehensive HIV/AIDS Partnerships (ACHAP) reported that Gaborone city had 880 and 750 while Francistown city 432 and 350 TB cases registered in ACHAP supported districts in 2012 and 2013 respectively [10]. According to the Ministry of Health [7], Gaborone and Francistown had the highest numbers of TB patients registered in СТВC in Botswana.

We conducted non-probably sampling in the qualitative phase, where respondents were selected by non-random methods, and not every person had an equal opportunity to be included in the sample. We performed convenience sampling because numerous respondents had missing contact information thus making it difficult to find them. Hence, a convenience sample was use for the readily available respondents. We determined the sample size for qualitative phase by data saturation, when no more new information was identified. Therefore, we observed data redundancy after forty (40) participants. We reviewed their clinical documents to identify patients with $\mathrm{TB}$ and interviewed them. A convenience sampling method was used for both Qualitative and quantitative designs. We also conducted non-probability sampling in the quantitative phase, where files were selected by non-random methods. A non-probability sampling design was used to select the sample. Moreover, we performed convenience sampling because several files were missing and difficult to locate. Hence convenience sample was use for the readily available files. In the quantitative phase, $670 \mathrm{~TB}$ records from the past 2 years (July 2016-Dec 2018) met the inclusion criteria and were extracted.

\subsection{Target Population}

The target population for the qualitative phase were all community members in СТBC who met the following inclusion criteria: 1) patient with TB registered with СТВС program during the study time frame (prospectively) and 2) Individuals, family members or relatives above 18 years of age with a TB patient at home or engaged in community TB care. The exclusion criteria included: 1) TB patients not registered with СТВC program and Individuals, family members or relatives with a TB patient at home not registered with CTBC and 2) TB Patients and their caregivers outside the study timeframe.

For the quantitative phase, records which met the inclusion criteria: 1) records of patients with TB registered with CTBC program prospectively or during the past two years before the study started (prospectively and retrospectively) and 2) records for male or female TB patients above 18 years of age. The exclusion criteria included: 1) records for TB patients not registered with the CTBC program, and 2) records for TB Patients outside the study timeframe. 


\subsection{Data Collection Methods and Procedures}

In the qualitative phase, we used an in-depth interview guide with open-ended questions guided by Roy's Adaptive Model (RAM) and Health Promotion Model (PHM) [11] to identify the community perceived factors contributing to effective active case findings and the community referral system. The interview guide had an observational tool that was used to record the caregiver's reaction during the interviews. The interviews were face to face at the participants' home after obtaining permission to write notes while they were answering. The interviews were conducted in English and Setswana. The language used depended on the participant's preference. The responses were documented in their own language to obtain complete information and reduce interviewer's biases of personal views and misinterpretation. The principal investigator and researcher thereafter translated the responses to English during analyses. All interviews were conducted by trained field workers comprising two clinical nurse specialists, past TB/HIV recruitment and retention officers and fourth year university bachelors' degree students who signed the confidentiality agreement after training $(\mathrm{n}=2$ each). The field workers were selected based on their experiences. Data collection commenced from July 04, 2018 to December 14, 2018.

A questionnaire with closed-ended questions was used in the quantitative phase to extract data from records of patients with $\mathrm{TB}$, who were registered with CTBC and met the inclusion criteria, prospectively and retrospectively, 2 years before the initiation of the study (July 2016). The data extraction began by identifying CTBC registered patients within the study time frame. The TB records were then separated from the remaining clinic records, followed by data extraction. During the data extraction, the clinic TB focal person was present in case the researcher/ field workers needed more clarification. For example, if the researcher/field workers required access to the electronic TB registers for verification of information. The researcher/field workers informed the clinic staff that they had almost completed the data extraction. Similar field workers collected data for both phases. We pilot tested the instruments to confirm the credibility and establish the validity and reliability.

\subsection{Data Analysis}

In qualitative phase, data analysis occurred concurrently with data collection to determine when data has reached point of saturation when no new information was identified. We divided the data into two sections as follows: 1) descriptive statistics for demographic data and 2) narrative data on perceived factors subjected to content analysis by naming and categorizing of phenomena through closely examination.

All the interviews were coded using an inductive approach and thematic analysis. This involved line-by-line analysis of each interview, naming each line and segment of data, grouping categories and contents of the categories, and also identifying deviant case, which were thereafter assembled into prominent themes. The 
transcripts and field notes were repeatedly read, analysed and initial thoughts were noted for each participant. Data from the in-depth interviews and reflective engagements are presented through quotes. The field notes from observations were utilised to inform and confirm the three themes that were identified during data analysis. To ensure validity and trustworthiness of the results of this qualitative study, the researcher prolonged the engagements with the participants by using in-depth interviews and asking probing questions to ensure long contact with the respondents. The research team read the transcripts several times, compared their results, and this led to the emergence of themes and subthemes as a measure of ensuring trustworthiness of the data. During the writing of the draft article, the emergent themes were compared with the transcripts individually and as a whole, while the findings from the observations and field notes were used to validate the interviews, which promoted reflexivity. The themes were categorized according to the components provided by RAM and HPM and aligned to answer the research questions of this phase.

We analysed quantitative data using STATA 14.0 Copyright 1985-2015 StataCorp LP [12]. Data was code according to the different variables under study and imported from an Excel spreadsheet into a STATA version 14.0 package. We used a fixed format to calculate the exact binomial confidence interval. Moreover, we calculated the odds ratio and compared between the sites under study. A comparison was performed between some study variables to identify the difference between two or more variables collected from the above mentioned two cities. We used descriptive statistics to describe the value attached to the contributions identified in this study through proportions.

\subsection{Ethical Considerations}

We obtained permission from the UNISA ethical committee, the Botswana Ministry of Health and Wellness, Health Research Development Committee and the participating sites before data was collected. All enrolled patients provided written informed consent. Confidentiality and anonymity were ensured by protecting the participants' identity and privacy. Participants were informed of their right to withdraw from the study at any time.

\section{Results}

\subsection{Qualitative Phase}

A total of 40 caregivers/treatment supporters were interviewed in this phase. The demographic characteristics provide personal information of the respondents on their age, gender, marital status, the level of education, employment status, religious affiliation, and their relationship to the patients. We analysed the demographics characteristics using graphs, frequencies, and percentages in Figure 1.

The interview and observation guides were based on the core categories of RAM namely the stimuli and the adaption and the HPM, which were individual characteristics and experiences resulting in behaviour outcomes. 


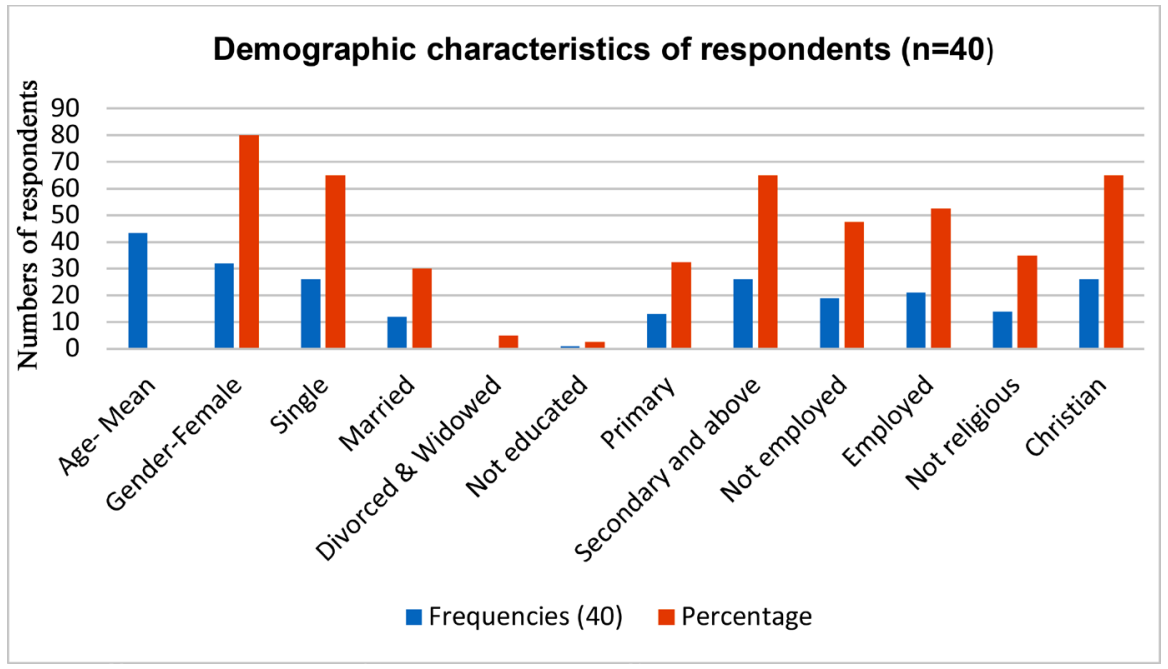

Figure 1. Demographic characteristics of respondents $(n=40)$.

\subsection{Analysis of the Adaptive Modes and Levels within RAM Levels and Behavioural Outcome in HPM}

Responses can either be "adaptive" and "effective" when human were coping with and meeting the terms of survival and growth of the system. In contrast, a failure to cope with and met the survival and growth expectation of the system can lead to "ineffective" responses. We identified positive adaptation from the major themes that emerged in this study.

The following major themes emerged from the data for effective contributing factors:

- Accepting to participate in СТВC,

- Health workers working as a team,

- Understanding their role as caregivers/treatment supporters,

- Communication between the health workers team and caregivers/treatment supporters and or patients,

- Caregivers/treatment supporters receiving health education on TB,

- Social and physiological Support for the patient and caregivers/treatment supporters.

\subsubsection{Self-Concept}

Self-concept mode is related to the psychological aspect of the individual or personal self, and how a person/individual behaves at a given time. Self-concept highlighted the effective (positive adaptation) or ineffective (negative adaptation) factors contributing to active case findings in CTBC. Given below is a discussion of the identified themes related to self-concept which indicated positive adaptation [11].

\subsubsection{Accepting to Participate in CTBC}

The acceptance of a caregiving role was one of the themes that emerged repeatedly within the interview, under different questions and for effective adaptation. Their acceptance to participate in CTBC was a sign of adaptation. 
The responses for indicating effective contributions of active findings in CTBC when inquired about incidences are provided below:

"It is for the caregiver and patient to accept the condition and be ready to assist the patient" (caregiver 26).

Thirty-three respondents (83\%) seemly accepted the role of caregiving and their contributions to active case findings regardless of its duties and did not experience any incident that they could be shared:

"Nothing/no incident" (caregivers 2, 4, 5, 6, 7, 9, 12, 17, 18, 20, 22, 27, 28, $29,31,34,35,38,39,40$ etc.).

\subsubsection{Understanding Their Role as Caregivers/Treatment Supporters} At least $20 \%$ (8) of the respondents indicated accepting the situation and understood the role in caregiving.

One such response is provided below:

"Understanding the role involved between supporter and nurses/other health Workers" (caregivers 2, 4, 5).

\subsubsection{Role Function}

This mode addresses how individuals socialize with each other to maintain order or fulfil expected responsibilities [11]. Given below is a discussion of themes that emerged in relation to the role function and showed positive adaptation.

\subsubsection{Health Workers Working as a Team}

Health workers working as a team were another theme that emerged under the contributing factors. Responses of $20 \%$ (8) of the caregivers were as follows:

"The teamwork in the clinic and when asking for help it is given immediately" (caregiver 3,4 ).

"Really helpful, every time you have a need, it is always addressed" (caregiver 7).

Another respondent who appeared to face difficulties with the caregiving role said,

"Health Education Assistant and a Social worker visited, I am not sure how often, but they come, maybe once a week" (caregiver 23).

\subsubsection{Caregivers/Treatment Supporters Receiving Health Education on} TB

Caregivers/treatment supporters receiving health education on TB was also another theme that emerged from at least $23 \%$ (10) of the responses under factors contributing to effective active case finding in CTBC.

One respondent said the following:

"Had enough information" (caregiver 19).

Another respondent also mentioned: 
"Health education about TB especially how to prevent TB from spreading to others" (caregiver 20).

Two other responses are provided below:

"Health education and checking the patient" (caregiver 31).

"Always available to tell them to assist with more education to the patient and caregiver" (caregiver 11).

\subsubsection{Interdependence}

Interdependence mode addresses adaptive behavior expected from both individuals and groups from their interdependent relationships while they provide and receive love, respect, support and value from their families, relatives, friends, and colleagues [11]. This mode also supported the role function mode, however at a broader aspect, as it not only involves the caregiver but also others. The aforementioned mode also indicated positive adaptation.

\subsubsection{Communication between the Health Workers Team and Caregivers/Treatment Supporters and/or Patients}

Communication between the health workers team and caregivers/treatment supporters and or patients also emerged as a theme. Given below are the common statements of $30 \%$ (12) of the respondents:

"Good communication and understanding between patient and supporter" (caregivers 2, 4, 5).

Some respondents also said the following:

"Communication and respect between supporter and nurses/other health workers" (caregivers 1, 3, 4).

\subsubsection{Social and Physiological Support for the Patient and Caregivers/Treatment Supporters}

Social and physiological support is extremely important in a caregiving role as it involves the relationship between the caregiver/supporter and the patient. The presence of support influences the adaptation level of the system.

At least $50 \%$ (20) of the responses were as follows:

"For relative to assist me in taking care of the patient" (caregiver 26).

"To have a woman help me" (caregiver 23).

Some respondents also said the following:

"Support for the sake of the patient during treatment Period" (caregiver 38, $30,32)$.

"Masks and gloves to help prevent the transmission of TB" (caregiver 24).

"Food supplements for the patient" (caregiver 16, 27).

"To be taking used nappies frequently, at least daily than delaying" (caregiver 23).

"Only food, that is, m/meal and beans" (caregiver 24). 


\subsection{Quantitative}

\subsubsection{CTBC Referral System}

Table 1 reports that men were affected more by TB than women as indicated by $54 \%$ of men on TB treatment compared to $46 \%$ of women.

A total of 670 patients were enrolled in this phase. The CTBC referral was investigated to determine the community referral system, i.e., how the caregivers'/ treatment supporters and patients were introduced into СТВC. This helped us identify the impact of their introduction on their adaptation to CTBC. The target population were presented with some questions to determine the adaptation level as results presented in Figure 2.

Figure 3 reports that all patients who opted for CTBC were registered with community care. However, while $58 \%$ of the caregivers had registered their names, $55 \%$ of contact details were recorded in the TB records. At least $65 \%$ close contacts who were provided referral letters for TB screening. Moreover, $18 \%$ of the patients screened positive for $\mathrm{TB}$, thus necessitating interventions for improvement

Table 2 demonstrates better performance of Gaborone than Francistown (81.9\% and $18.1 \%$ respectively), in terms of $\mathrm{TB}$ suspect/contacts referral letters and the investigation of those screened positive for TB ( $83.3 \%$ vs $16.7 \%$, respectively). The differences were significant $(p$-value $=0.001)$.

\subsubsection{Active Case Finding and Contacts Screening}

Active case finding and contacts screening are important aspects of TB control.

Table 1. Demographic characteristics.

\begin{tabular}{cccc}
\hline Characteristics & Total population & Proportion (\%) & 95\% CI \\
\hline Age $<35$ years & $261 / 670$ & 61 & $57-65$ \\
Gender - Female & $311 / 670$ & 46 & $43-50$ \\
\hline
\end{tabular}

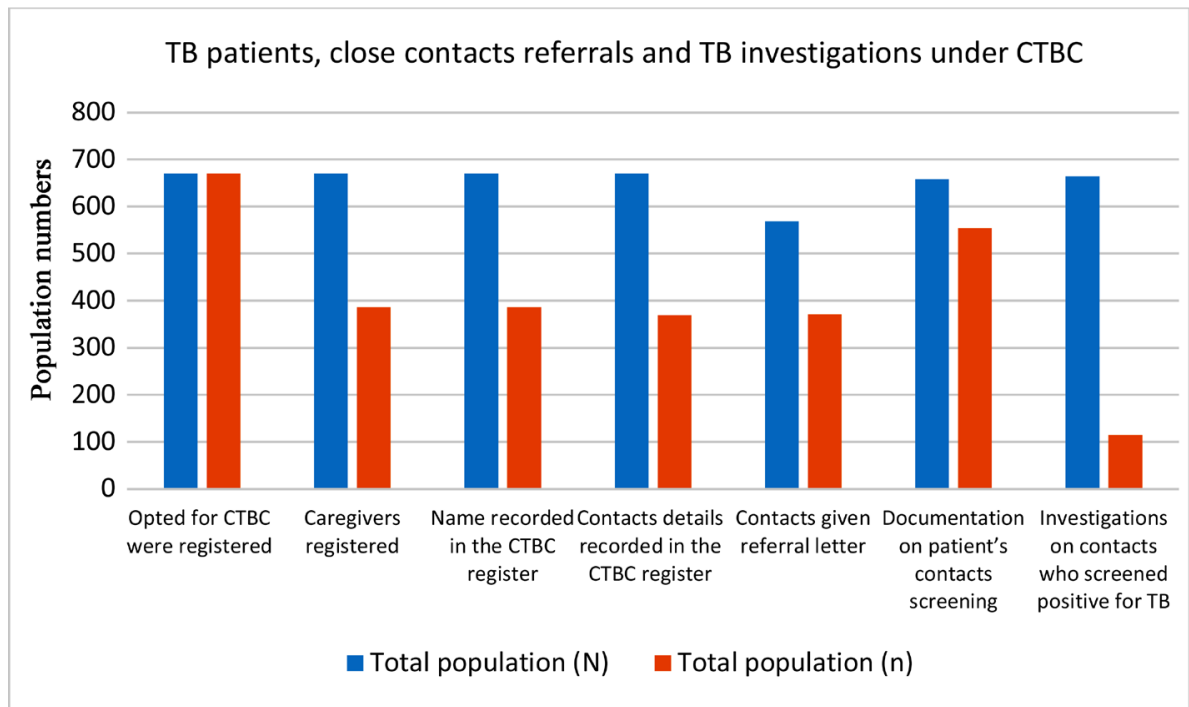

Figure 2. TB patients, close contacts referrals and TB investigation under CTBC. 


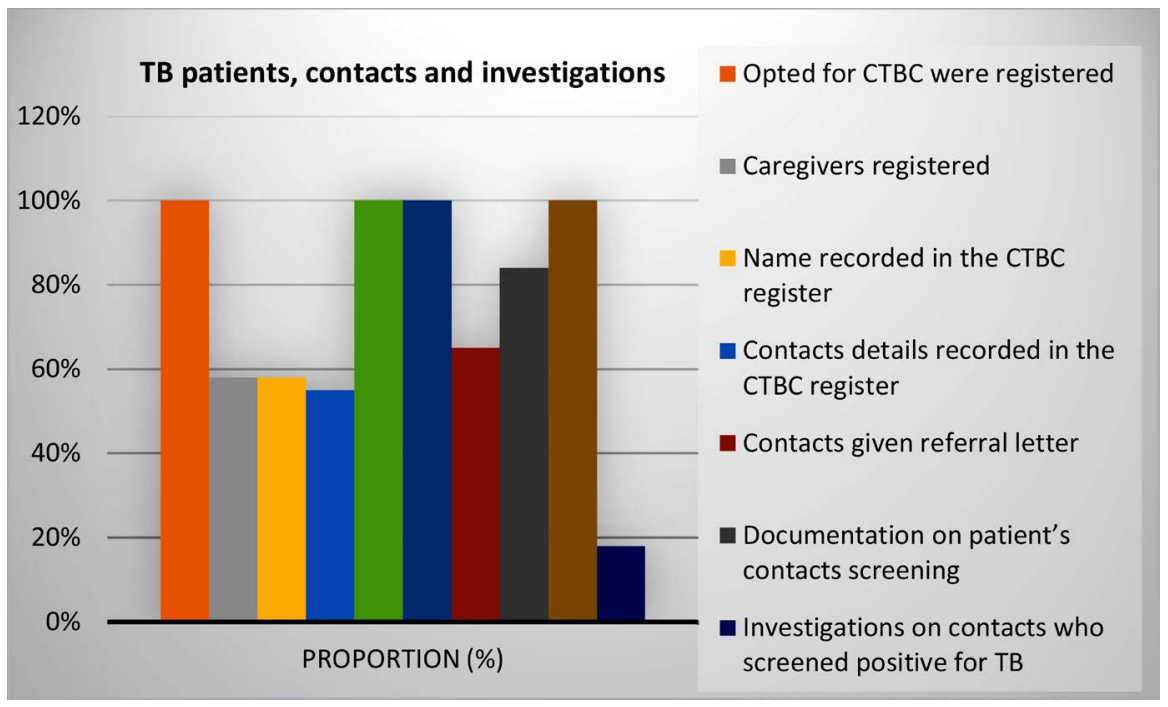

Figure 3. Proportions of TB patients, close contacts referrals and TB investigation under CTBC.

Table 2. TB suspect/contact referral comparison between Francistown and Gaborone.

\begin{tabular}{cccccc}
\hline TB suspect/contact Referral & $\begin{array}{c}\text { Sites for the study=n (\%) } \\
\text { Francistown Gaborone }\end{array}$ & OR & (95\% CI) & P-value \\
\hline $\begin{array}{c}\text { TB Patient's suspect/contacts } \\
\text { referred with a letter } \\
\begin{array}{c}\text { Patient's documented } \\
\text { suspect contacts screened }\end{array}\end{array}$ & $67(18.1)$ & $304(81.9)$ & 6.55 & $4.45-9.65$ & 0.001 \\
\hline $\begin{array}{c}\text { TB Investigations } \\
\text { Investigations done on } \\
\text { patient's contacts who } \\
\text { screened positive for TB }\end{array}$ & $20(16.7)$ & $310(56)$ & 0.72 & $0.47-1.10$ & 0.127 \\
\hline
\end{tabular}

Figure 4 depicts active case finding and contact screening in СТВC and the contribution of the community in identifying TB cases. While $94 \%$ (1311/1398) active case finding suspects/contacts had been screened for TB, only 10\% (134/ 1311) suspects/contacts screened positive. Of those positive patients, $18 \%$ (115/ 1398) were referred for investigated. Moreover, $89 \%$ (102/1398) had their results followed-up. We obtained 3\% (35/1398) TB positive results, following which all patients underwent treatment. We observed low active case findings from the screening stage until their enrollment in the treatment. The treatment success was low at $71 \%(25 / 35)$ with $3 \%(1 / 35)$ death and $26 \%(9 / 35)$ being transferred out and having missing information.

\section{Discussion}

Our results reported that men were more affected by TB than women (54\% vs $46 \%$ ) respectively (Table 1 ). This was similar to a report on the evaluation of community. TB approaches in Botswana were $54 \%$ and $46 \%$ of the study cohort that were men and women, respectively [7]. Caregivers had a mean age of 43.4 


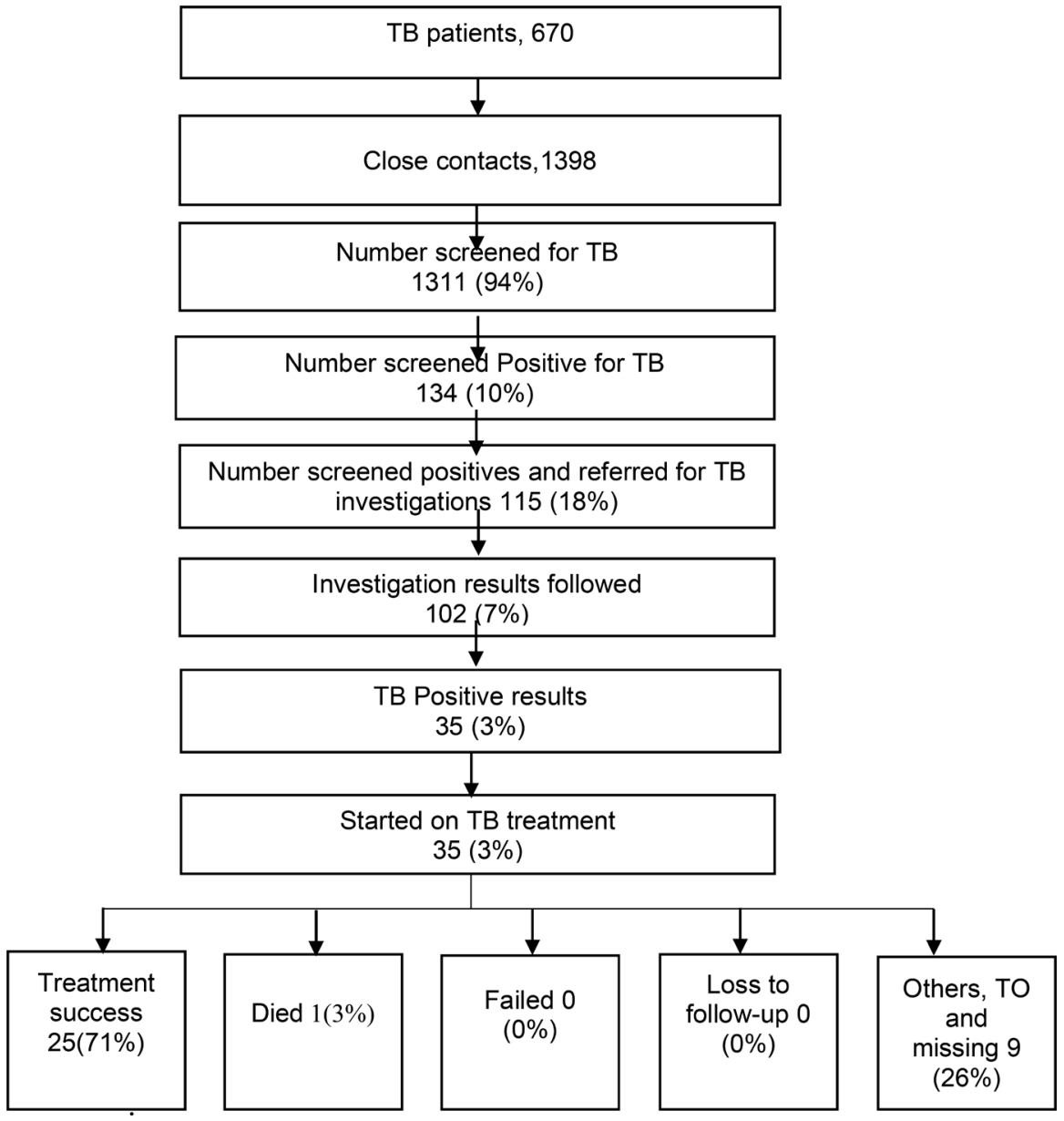

Figure 4. Active case finding and close contacts screening.

years, comprising young adults who were still active in society and a lot of them being $(80 \%)$ women. This result concurred with the statistical findings that indicated Botswana was a female-dominated society [9]. Statistics Botswana has also identified a trend in which the proportion of single women was increasing and becoming higher than men, with a higher percentage of women engaged in no paying jobs, such as caregiving. The aforementioned trends were local regional and global [9].

Our results reported on the low effects of community TB caregivers' contribution towards active case findings through contact tracing. All patients who opted for CTBC (100\%) were registered into СТВC. However, only 55\% of the caregivers had their names and contact details recorded in the patients' $\mathrm{TB}$ records during CTBC referral to enable active case findings. The caregivers in CTBC were responsible for assisting in active case findings of TB contacts. This can be attributed to their regular contact with the patients, but the absence of contacts details interfered with numbers of contacts being investigated on TB infection hence reducing the number of presumptive TB. Active case findings of TB were the most effective strategy to help identify TB cases [1] [4] [13] [14] [15]. We observed missing information on the contacts details which negatively contrib- 
uted to low numbers of contact screening and tracing. This eventually necessitated continuous quality improvement activities, training on data quality and reporting to improve information on contact details so as to increase number of contact screening, treatment outcome and general TB management [16].

Roughly half of the caregivers and at least two-thirds TB patients' close contacts were provided referral letters for TB screening, thus excluding one-third of close contacts. Hence, the presumptive TB cases were missing. Of the aforementioned, two-thirds referred for screening, $83 \%$ of TB close contacts had relevant screening documentation, thereby eliminating additional presumptive TB cases. Our data on CTBC referral for close contacts were consistent with the results of a study strengthening referral system in the community health programs. The study also high-lighted barriers in the use of referral data, resulting in lack of feedback to enable optimal functioning of the referral system and the need for improving access to TB care and case detection [16] [17] [18]. Results of a systematic review of active case finding strategies for $\mathrm{TB}$ in the homeless population were in agreement with our findings on the issues of community referral. It recommended modifications in the referral pathways for improvement, considering evidences for weak professional support and education uptake [19].

The theme acceptance repeatedly emerged during the interviews. According to the Botswana community TB care policy guidelines (2011), all patients with TB must be provided the option of deciding if they want to join CTBC. The acceptance of role by caregivers indicated the community was happy and felt motivated by their role in providing services to fellow community members [4] [20]. Moreover, the presence of Social and physiological support is critical in caregiving roles, because they contribute to a substantial number of case findings if implemented effectively. [4] [13] [21] [22] [23].

Health education facilitates the empowerment patients, caregivers, family members, and community and must be provided at all levels of TB treatment using effective health education strategies, including training for better understanding of TB. Imparting health education on TB to caregivers/treatment supporters receiving health education on TB was also another theme that emerged from at least $23 \%$ (10) of the respondents. Our findings reveal that only a quarter of the caregivers received health education to enable behaviour change. This in turn indicated the unaddressed needs and expectations of the community, such as support and education [2] [3] [4] [20] [22] [24] [25]. According to WHO, health education is a principle by which individuals, communities, a group of people in any learning experience, learn to behave in a way that is influenced by their knowledge to help them improve their health. Health education has been identified as a tool that can be used effectively to improve health as it can re-shape people's unhealthy lifestyles to health behaviour.

We recommend the development of different health education strategies e.g., booklets or pamphlets, posters, face to face, group discussions and videos for patients under СТВC and their caregivers/treatment supporters with a feedback 
loop. Moreover, we recommend structured community supportive supervision and referral process with follow-up and a feedback loop for the patients and caregivers, the development СТВC training modules and standard operating procedures or algorithms including community referral.

Our study had some limitations in that it could have focused on all patients with their families, relatives, friends and community volunteers in community TB care. However, we could not cover the entire population because of the widely spread health facilities in the country, thereby limiting the generalizability of results. Owing to age restrictions, we failed to include some patients and their supporters. This resulted in missing important information on patients with TB and their caregivers.

In conclusion, community TB care exerted marginal effects on the early identification of TB referral, infected cases, active case findings and contact screening. This necessitates extensive improvement in the area of health education and referral system for improving CTBC and reducing TB.

\section{Acknowledgements}

We are grateful to all caregivers and patients who participated in the study. We thank the field workers teams for data collection. Our sincere gratitude also goes to health care workers at all clinics which participated for their cooperation and assistance. Finally, we are also grateful to the Botswana Ministry of Health and Wellness, District Management Health Teams and clinics for their participation and support.

\section{Conflicts of Interest}

The authors declare no conflicts of interest regarding the publication of this paper.

\section{References}

[1] WHO World Health Organization Website. Global Tuberculosis Report 2019. http://www.who.int/teams/global-tuberculosis-programme/tb-reports

[2] WHO World Health Organization Website. Global Tuberculosis Report 2017. http://www.who.int/teams/global-tuberculosis-programme/tb-reports

[3] Ministry of Health (of Botswana) (2018) National Tuberculosis Control Programme Manual. Eighth Edition, Government Printers, Gaborone.

[4] Ministry of Health (of Botswana) (2011) National Tuberculosis Programme: (BNTP) Botswana Community Tuberculosis Care Policy Guidelines. Government Printer, Gaborone.

[5] World Health Organization. Global Tuberculosis Report 2015. http://www.who.int/teams/global-tuberculosis-programme/tb-reports

[6] World Health Organization. The End TB Strategy: Global Strategy and Targets for Tuberculosis Prevention, Care and Control after 2015. WHO, Geneva.

[7] Ministry of Health (of Botswana) (2012-2013) Report on Evaluation of Community Tuberculosis Approaches in Botswana. Government Printers, Gaborone. 
[8] Ministry of Health (of Botswana). National Tuberculosis Program Strategic Plan 20132017. Government Printers, Gaborone.

[9] Statistics Botswana (2014) Population and Housing Census 2011: Analytic Report. Government Printers, Gaborone.

[10] African Comprehensive HIV/AIDS Partnerships. Annual Report, 2013. Partnerships for a Health Africa, Gaborone.

[11] George, J.B. (2011) Nursing Theories: The Bases for Professional Nursing Practice. 6th Edition, Pearson, Boston.

[12] StataCorp. Stata Statistical Software. Version 14.0 College Station, 2015. StataCorp LP, College Station.

[13] Okelloh, D., Achola, M., Opole, J., Ogwang, C., Agaya, J., Sifuna, P., et al. (2019) Lessons Learned from Community-Based Tuberculosis Case-Finding in Western Kenya. From International Union against Tuberculosis and Lung Disease.

[14] Karamagi, E., Sensalire, S., Muhire, M., Kisamba, H., Byabagambi, J., Rahimzai, M., et al. (2018) Improving Tuberculosis Notification in Northern Uganda: Evidence of a Quality Improvement Guided Active Case Finding Intervention. BMC Health Services Research, 18, 954. https://doi.org/10.1186/s12913-018-3786-2

[15] Quang Vo, L.N., Forse, R.J., Condlin, A.J., Vut, N., Leg, T., Do, G.C., et al. (2020) A Comparative Impact Evaluation of Two Human Resource Models for Community Based Active Tuberculosis Case Finding in Ho Chiminh City, Vietnam. BMC Public Health, 20, 934. https://doi.org/10.1186/s12889-020-09042-4

[16] Kleinman, N.J., Mawandia, S., Kgwaadira, B., Broz, J., Matumo, H., Moumakwa, R., et al. (2017) Increasing Tuberculosis Register Data Quality in Botswana with Continuous Quality Improvement Activities. Quality in Primary Care, 26, 45-48.

[17] Querri, A., Ohkado, A., Yoshimatsu, S., Coprada, L., Lopez, E., Medina, E., et al. (2017) Enhancing Tuberculosis Patient Detection and Care through Community Volunteers in the Urban Poor, the Philippines. Public Health Action, 7, 268-274. https://doi.org/10.5588/pha.17.0036

[18] Mohajer, N. and Singh, D. (2018) Factors Enabling Community Health Workers and Volunteers to Overcome Socio-Cultural Barriers to Behavior Change: Meta-Synthesis Using the Concept of Social Capital. Human Resources for Health, 16, 63.

https://www.ncbi.nlm.nih.gov/pubmed https://doi.org/10.1186/s12960-018-0331-7

[19] Give, C., Ndima, S., Steege, R., Ormel, H., McCollum, R., Theobald, S., et al. (2019) Strengthening Referral Systems in Community Health Programs: A Qualitative Study in Two Rural Districts of Maputo Province, Mozambique. BMC Health Services Research, 19, 263. https://www.ncbi.nlm.nih.gov/pmc/articles/PMC6489304 https://doi.org/10.1186/s12913-019-4076-3

[20] Hamilton, K., Tolfree, R. and Mytton (2018) A Systematic Review of Active CaseFinding Strategies for Tuberculosis in Homeless Population. International Journal Tuberculosis and Lung Diseases, 22, 1135-1144.

https://doi.org/10.5588/ijtld.17.0784

[21] Karunga, R.N., Mireku, M., Muturi, N., McCollum, R., Vallieres, F., Kumar, M., et al. (2019) Supportive Supervision of Close-to-Community Providers of Health Care: Finding from Action Research Conducted in Two Countries in Kenya. PLoS ONE, 14, e021644. https://doi.org/10.1371/journal.pone.0216444

[22] Yassin, M.A., Datiko, D.G., Tulloch, O., Markos, P., Aschalew, M., Shargie, E.B., et al. (2013) Innovative Community-Based Approaches Doubled Tuberculosis Case Notification and Improve Treatment Outcome in Southern Ethiopia. PLOS ONE, 8 , 
e63174. https://www.plosone.org

https://doi.org/10.1371/journal.pone.0063174

[23] Asseyo, R.E., Mumma, J., Scott, K., Nelima, D., Davis, E., Baker, K.K., et al. (2018) Realities and Experiences of Community Health Volunteers as Agents for Behaviour Change: Evidence from an Informal Urban Settlement in Kisumu, Kenya. Human Resources for Health, 16, 53. https://doi.org/10.1186/s12960-018-0318-4

[24] Lukman, M., Ibrahim, K., Yani, D.I., Sari, S.P. and Juniarti, N. (2019) Exploring Strategies to Improve the Performance of Community Health Volunteers for Tuberculosis Care and Prevention: A Qualitative Study. International Journal of Community Based Nursing \& Midwifery, 7, 270-278.

[25] Balogun, M., Sekoni, A., Meloni, S.T., Odukoya, O., Onajole, A., Longe-Peters, O., et al. (2015) Trained Community Volunteers Improve Tuberculosis Knowledge and Attitudes among Adults in a Periurban Community in Southwest Nigeria. The American Journal of Tropical Medicine and Hygiene, 92, 625-632.

https://doi.org/10.4269/ajtmh.14-0527 